\title{
Patch Antenna Array Fault Modeling and Its Monitoring
}

\author{
Umesh Kumar $^{1}$, Rajiv Kapoor ${ }^{2}$ \\ ${ }^{I}$ (Student, Delhi Technological University, India) \\ ${ }_{2}^{2}$ (Faculty, Delhi Technological University, India)
}

\begin{abstract}
The On/Off faults are among the most prominent hazards observed in antenna arrays. They behave like a catalyst towards steady degradation of the circuit's performance. This paper is regarding fault monitoring in the microstrip patch antenna array [1][6]. The fault modeling, included here is single and double fault models of the patch antenna array of 8 elements. There is a marked deviation in the parametric properties of the array because of On/Off faults. By observation and analysis of these trends, the detection of fault in the array is simplified to a great extent. We aim at identification of On/Off faults in a faulty array structure by observation of its characteristic properties (like S- parameters and Radiation Pattern) and their analysis using Matrix Comparison Method and Scaled Conjugate Gradient Back Propagation Algorithm in Artificial Neural Network.
\end{abstract}

Keywords: Patch antenna, patch array antenna feed, S-parameter, radiation pattern, characteristic impedance, neural network.

\section{INTRODUCTION}

Microstrip array [1],[2] is designed at $2.49 \mathrm{GHz}$ used in IEEE $802.11 \mathrm{~b}$ high-speed TCP/IP communications. It can be used as mass-produced, inexpensive equipment designed for FCC part 15 unlicensed uses. Microstrip antennas have been one of the most innovative topics in antenna theory and design in recent years, and are increasingly finding application in a wide range of modern microwave systems. The development of microstrip antennas has been driven by systems requirements for antennas with low-profile, low-weight, lowcost, easy integrability into arrays or with microwave integrated circuits, or polarization diversity. One of the best features of microstrip antennas is the ease with which they can be formed into arrays, and a wide variety of series-fed, corporate-fed, scanning, and polarization-agile arrays have been designed using microstrip elements. With the help of IE3D simulation tool, which is most versatile, easy to use, efficient and accurate electromagnetic simulation tool, the single patch as well as patch array has been simulated successfully. For modelling and simulation of Matrix Comparison Method [13] and Scaled Conjugate Gradient Back Propagation Algorithm in Artificial Neural Network [9], the MATLAB software is used.

\section{Design Of Single Patch}

The required specifications for the patch [9] are:

Resonant Frequency $\left(f_{0}\right)=2.49 \mathrm{GHz}$

Dielectric constant of Substrate $=2.33$

Height of the substrate $=1.6 \mathrm{~mm}$

Matching Load $=50 \Omega$

Using the standard formulae and the standard design considerations we obtain a single patch with following parameters:

Width of the patch $(W)=46.66 \mathrm{~mm}$

Effective Dielectric $\left(\epsilon_{\text {reff }}\right)=2.2248$

Effective Length $($ Leff $)=40.40 \mathrm{~mm}$
Length extension $(\Delta L)=0.8175 \mathrm{~mm}$

Length of the patch $(L)=38.765 \mathrm{~mm}$

Inset position $(y)=13.665 \mathrm{~mm}$

Inset width $\left(W_{0}\right)=5.2578 \mathrm{~mm}$

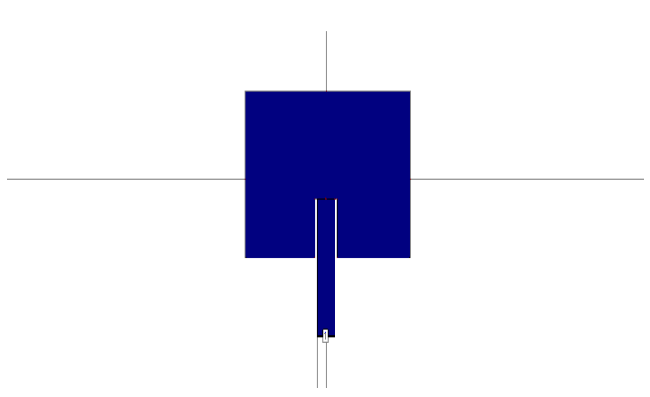

Fig. 1-schematic of single patch element with inset line feed 
Fig. 2- The reflection loss is around $-37.5 \mathrm{~dB}$ indicating effective coupling between port and element.

The microstrip inset feed line (figure 1) is a conducting strip, usually of much smaller width compared to the patch. It's easy to fabricate, simple to match by controlling the position of inset [3][4] and rather simple to model. But with increase in substrate thickness, spurious and surface radiation increase resulting in limiting the bandwidth.

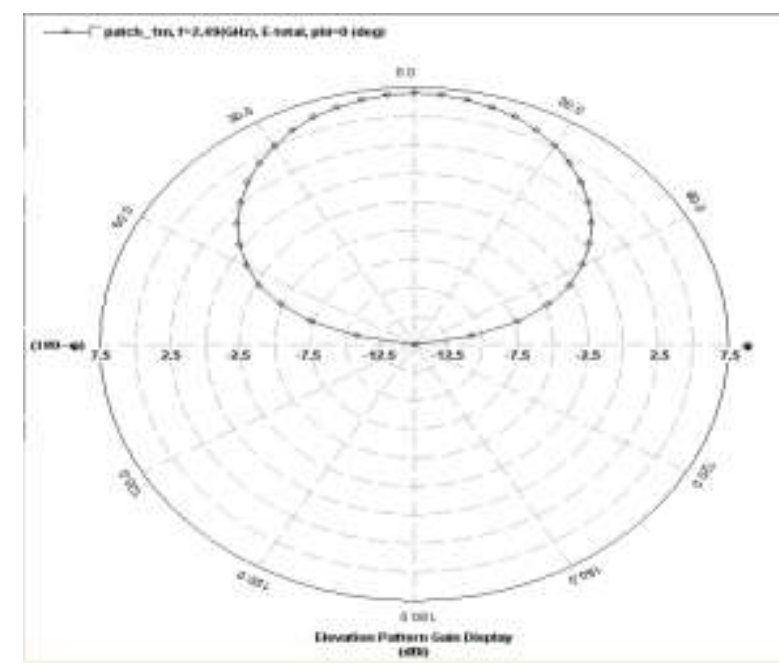

Fig. 3- This graph gives us the 2D view of radiation pattern in the azimuthal plane. The half power beamwidth is determined to be $74.42^{\circ}$.

We determine the physical spacing between the elements and the structure of the feed using the above information. To have zero grating lobes we kept the inter element spacing to be $\lambda / 2$. Since any larger distance resulted in one or more grating lobes while smaller distances led to introduction of inherent asymmetries into the design. The feed length was taken to be approximately $\lambda / 4$ for impedance transformation at the desired resonant frequency of $2.49 \mathrm{GHz}$. The feed width was chosen on a hit-and-trial basis to obtain the required load matching of $50 \Omega$. The initial width of the feed connecting the single element to the entire feed structure was taken to be the same as inset width $\left(\mathrm{W}_{0}\right)$.

\section{Antenna Array Fault Modeling}

The antenna array feed is based on parallel feed technique with improved beam shape [7]. The resonant frequency has changed from $2.49 \mathrm{GHz}$ to $2.5 \mathrm{GHz}$ (figure-2). This much of shift is tolerable since the maximum limit of tolerance is $2 \%$. The shift in our case is $0.43 \%$. As we can see that at resonant frequency we have a dip of $-37.5 \mathrm{~dB}$ which gives very good antenna performance (figure 5). Here we have considered only the single and double fault modelling with different combinations of faults in the elements of the patch antenna array. The deviation in values of reflection coefficient and radiation pattern from those of fault-free case is valid. At the resonance frequency, these deviations are reduced. Hence, the patch array is very less prone to single fault errors. In case of double faults, these deviations are much higher than those of single fault cases. At resonance frequency, these deviations got reduced. Although the deviations are within tolerable limit, they are still traceable.

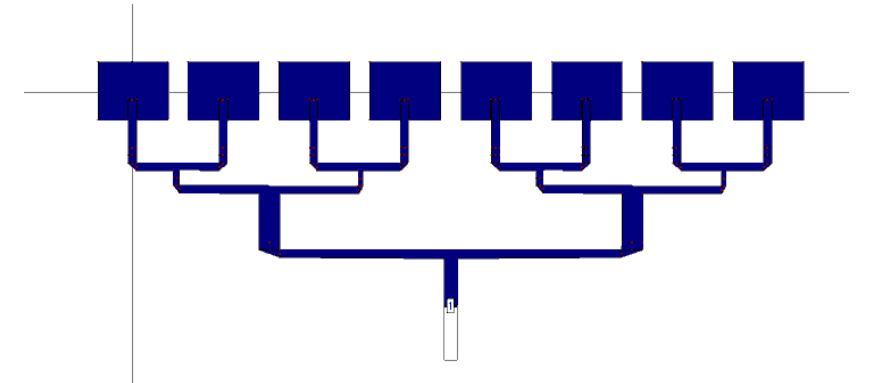

Fig. 4- schematic for 8 element array

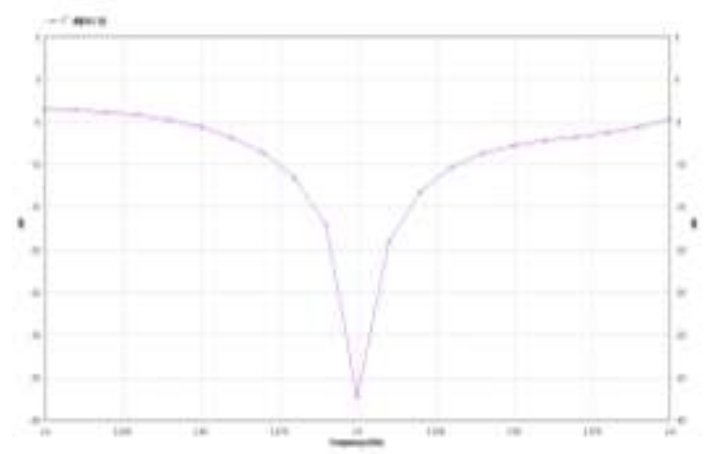

Fig. 5- Plot of $\mathrm{S}[1,1]$ in $\mathrm{dB}$ scale

The deviation in values of reflection coefficient and radiation pattern from those of fault-free case is valid. At the resonance frequency, these deviations are reduced. Hence, the patch array is very less prone to single fault errors. In case of double faults, these deviations are much higher than those of single fault cases. At resonance frequency, these deviations got reduced. Although the deviations are within tolerable limit, they are still traceable. 


\section{III a. The single fault}

In the following figures, the fault model is shown with feed discontinuity fault in the first element and the antenna characteristics parameter.

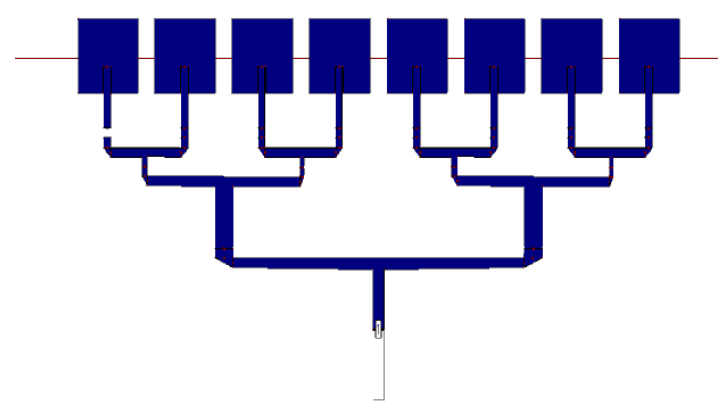

Fig. 6-discontinuity of feed in first patch element

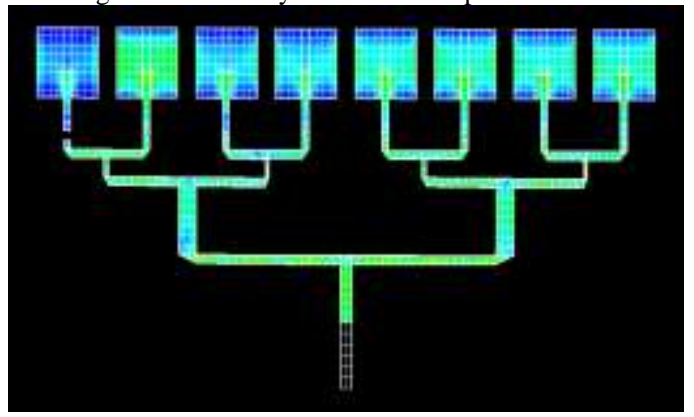

Fig. 7 - current distribution in single fault

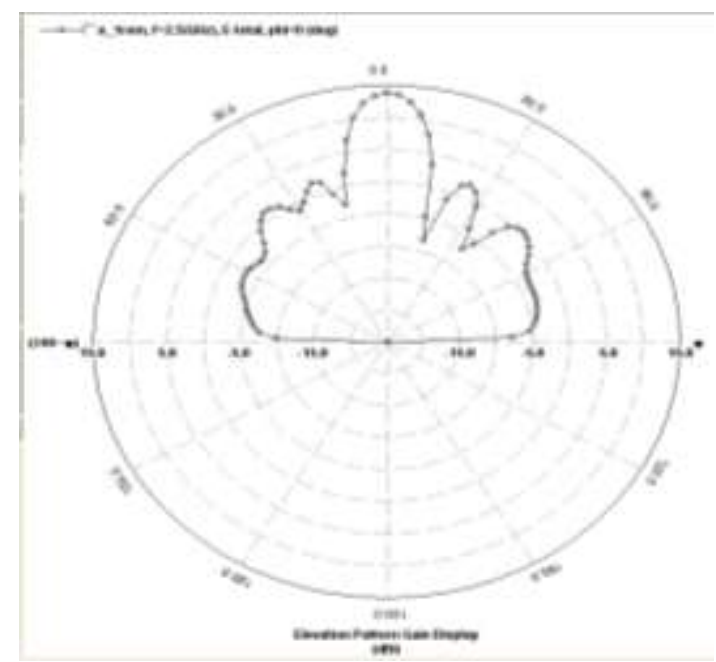

Fig. 8-2D polar plot of gain pattern

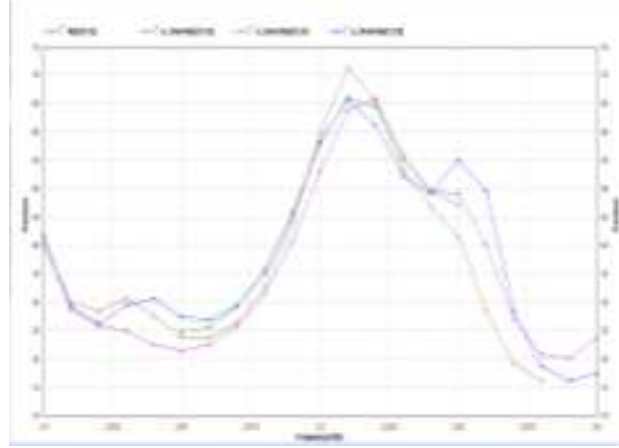

Fig. 9- $\operatorname{Re} \mathrm{Z}[1,1]$ for all combinations of faulty cases

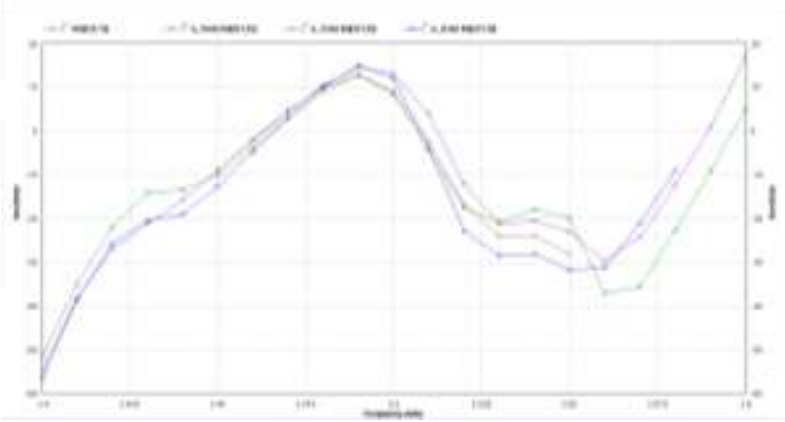

Fig. 10- Im Z[1,1] for all combinations of faulty cases

The point here is to be noted that patch with discontinued feeding is still showing current distribution, which is the parasitic effect [8] of the elements nearby.

\section{III b. The double fault}

In the following figures, the fault model is shown with feed discontinuity fault in the first and second element and the antenna characteristics parameter. 


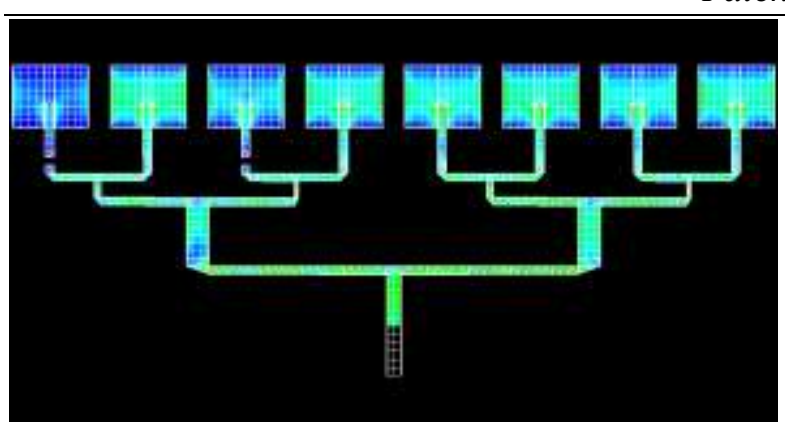

Fig. 11 - current distribution in double fault

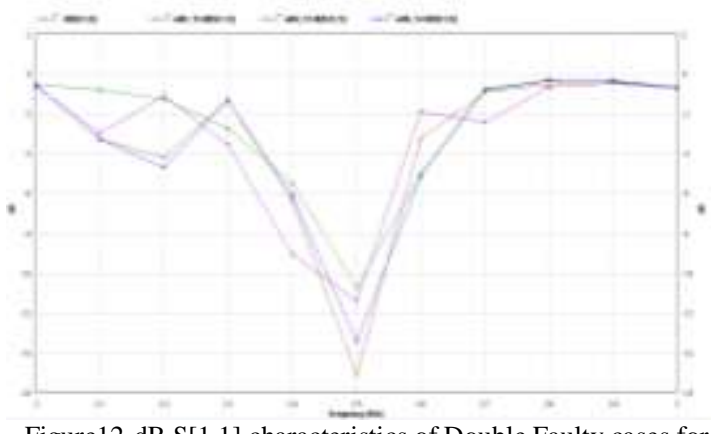

Figure12-dB S[1,1] characteristics of Double Faulty cases for combinations of $(1,3),(1,4)$ and $(1,5)$ faults

In the following figures antenna characteristics for the fault of combinations of $(1,3),(1,4)$ and $(1,5)$ are only shown (figure 12,13). Rest of the cases can similarly be modelled.

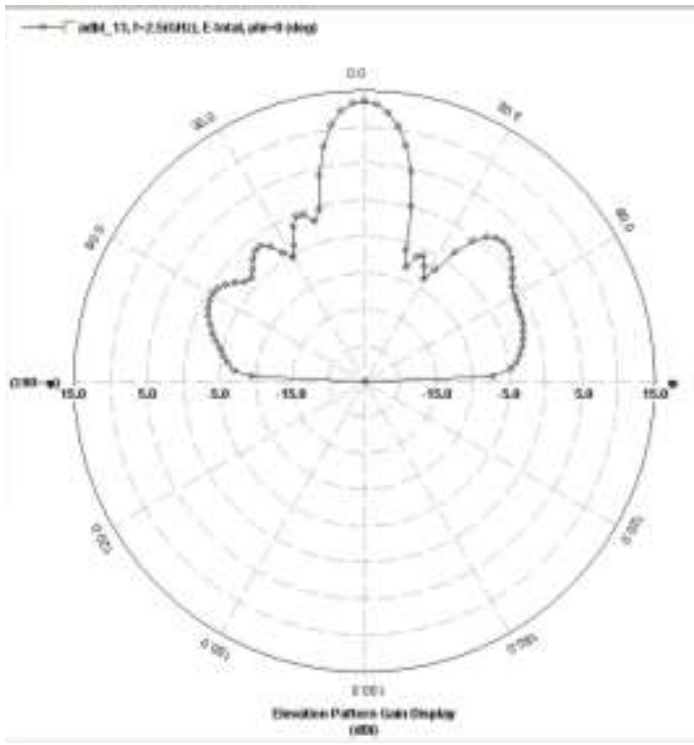

(a)

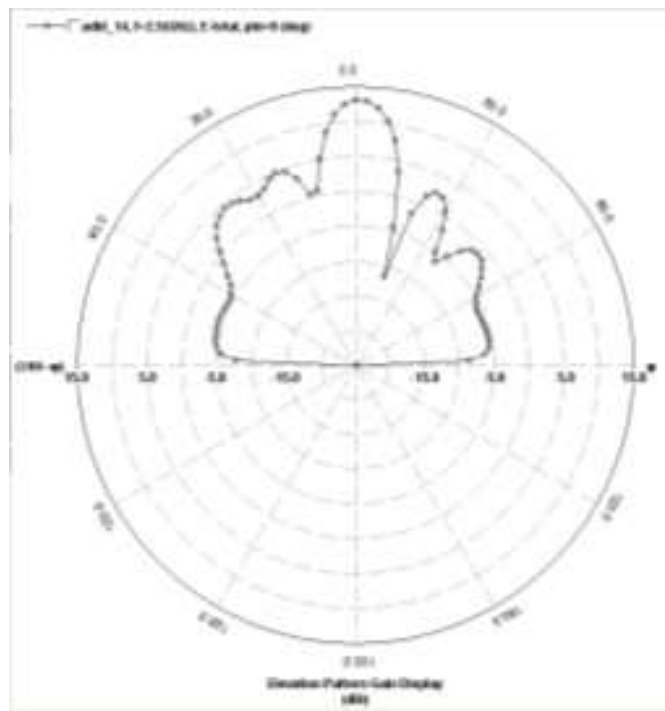

(b)

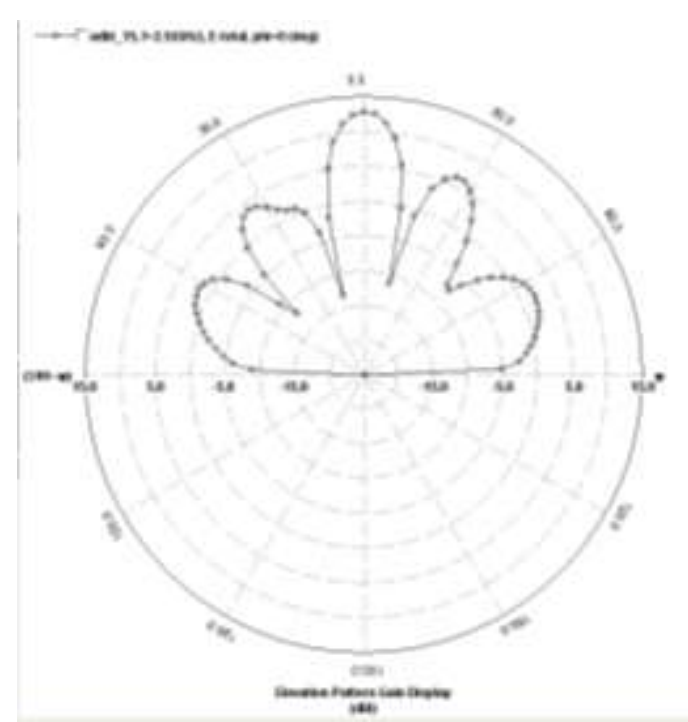

(c)

Figure 13-2D polar plot of Double Faulty cases for combinations of a $(1,3), b(1,4)$ andc $(1,5)$ faults

These are the 2D polar plots for each of the double faulty cases, which shows gain for double fault cases of $13.35 \mathrm{dBs}$. 


\section{IV a. Matrix Comparison Method}

\section{Antenna Array Fault Monitoring}

The efficiency of the matrix comparison method [13] for the fault identification is up to $85 \%$ on an average for up to double fault in array.

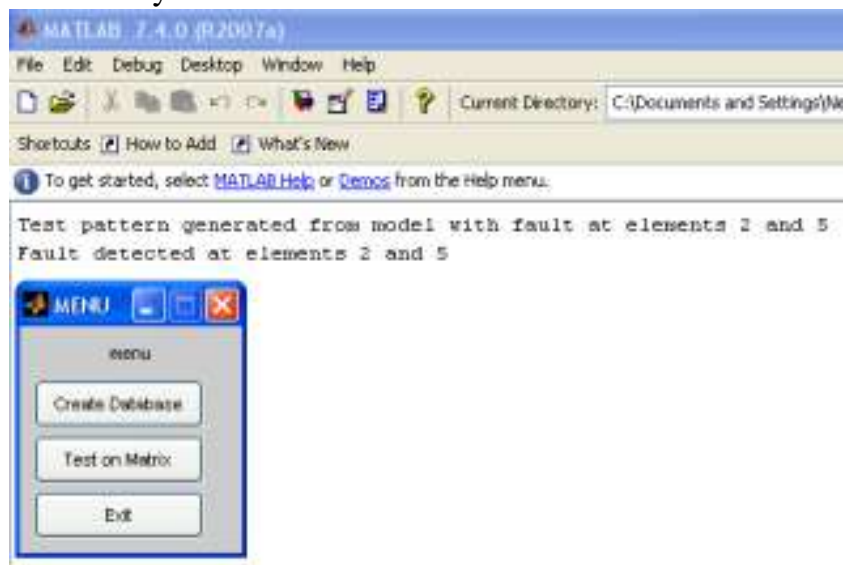

Fig.14- simulation of matrix comparison method with MATLAB

\section{IV b. Artificial Neural Network Method}

The ANN is based on Scaled Conjugate Gradient Back Propagation Algorithm in Artificial Neural Network [14][15], gives an accuracy of more than $95 \%$ and more for most of the test cases.
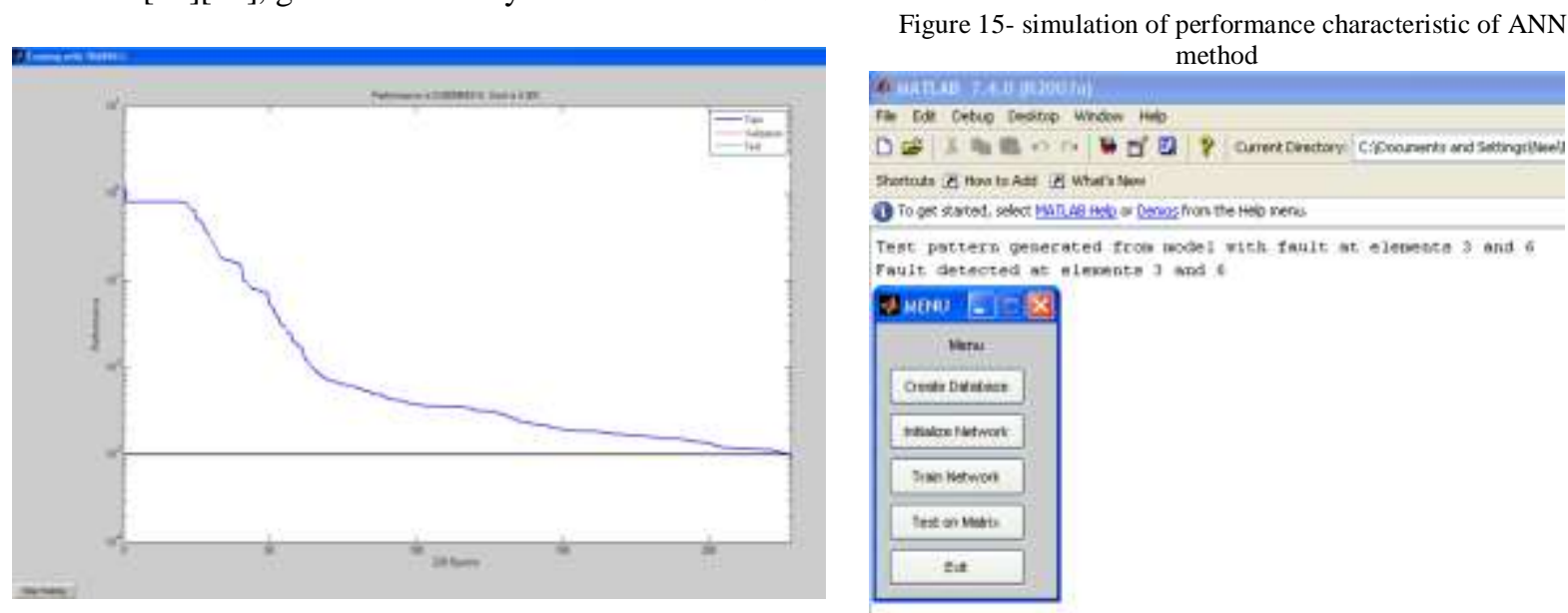

Figure 16- fault monitoring simulation

Monitoring these deviations using ANN(figure 15) we obtain the following Neural Network training statistics:

Epochs elapsed $=228$

Time elapsed $=2$ min $6 \mathrm{sec}$

Performance achieved $=0.000964314$

Gradient at last $=0.0326946$

\section{Conclusion}

The design of patch antenna for IEEE 802.11 has been successfully implemented at $2.49 \mathrm{GHz}$. The patch gives us very accurate results.

The 8 - element microstrip antenna array meets the requisite specifications. Although there is relative shift in resonant frequency (from 2.49 to $2.5 \mathrm{GHz}$ ), it is within tolerable limits (2\%).

It is found out that on an average the Matrix Comparison Method gives an accuracy of $80-85 \%$. This method is also found to be susceptible to ambiguity decisions.

The ANN gives an accuracy of more than $95 \%$ and more for most of the test cases. This method is free of any error generated because of ambiguity.

\section{Future Scope}

Dipole antennas [12] can be used instead of microstrip patches. It is known that dipoles are more susceptible to flaws in array structures and hence errors can be easily traced. Circularly polarized patches can be used instead of linearly polarized ones. Cavity model analysis can be used instead of transmission line model. 
The same analysis can be implemented for a larger array structure consisting of 32/64 elements. Higher degree of faults can be analysed in the array.

\section{References}

[1] H. Entschladen, U. Nagel ,"Microstrip patch array antenna”,IEEE Trans.1984,PP. 931-933.

[2] K.A. Carver and J.A. Mink, "Microstrip antenna technology", IEEE transactions on Antennas \& Propagation, VOL. 29, January 1981, PP. 2-24.

[3] Edward H. Newman, John E. Tehan, “Analysis of microstrip arrya and feed network”,IEEE Trans. VOL. AP-33,1985.

[4] M. Ramesh AND Yip KB ," Desing formula for inset fed microstrip patch antenna ", journal of microwave and optoelectronics VOL. 3 N. Dec-2003.

[5] N. Bayat, H. R. Hassani, S. Mohammad and Ali Nezhad, "Sidelobe reduction in microstrip patch antenna array", IEEE Trans. NOV2011

[6] J.R. James, P.S. Hall, 'Handbook OF Microstrip Antennas' VOL. 2, Peter Peregrinus ltd., London United Kingdom, 1989.

[7] A. Mohd. Tarmizi, M.T. Mohd Wor, A.R. Tharek and R. Abdolee,'Reconfigurable Microstrip Patch Antenna Array with Beam Shaping" IEEE TRANS.2007

[8] Shuguang Chen and Ryuichi Iwata,"Mutual Coupling Effect in Microstrip Patch Array Antenna" IEEE TRANS 1998.

[9] Z. I. Dafalla, W.T.Y. Kuan, A.M. Abdel Rahman and S.C. Shudakar,"design of a Rectangular Microstrip Patch Antenna at 1 GHZ" IEEE Trans, RF and microwave confrence oct. 2004.

[10] QI-JUN Zhang, Kuldip C. Gupta,Vijay K. Devabhaktuni, “Artificial Neural Networks for RF and Microwave Design: From theory to practice".

[11] Abhilasha Mishra, A.B. Nand Gaonkar, V.D. Bhagile, S.C. Mehrotra, P.M. Patil," "Design OF Square AND Rectangular Microstrip antenna with the use of FFBP algorithm of artificial neural network" IEEE TRANS. 2009.

[12] Huiling Jiang; Fumio Kira; Keizo Cho. "Printed Dipole Antenna Comprising Partially Cylindrical Parasitic Element" Microwave Conference proceedings, 2005. APMC 2005. Asia-pacific conference proceedings VOLUME: 3 .

[13] Ming-TE Liang, Chin-Ming Lin, and Chi-Jang Yeh," Comparison Matrix Method and its Applications to Damage Evaluation for Existing Reinforced Concrete Bridges" journal of marine science and technology, VOL. 11, NO. 2, PP. 70-82 (2003).

[14] Orlando De Jesús AND Martin T. Hagan, “backpropagation algorithms for a broad class of dynamic networks” IEEE Trans, March 2006.

[15] Xiao-Hu Yu, Guo-An Chen, Shi-Xin Cheng," Dynamic Learning Rate Optimization of the backpropagation algorithm” IEEE Transactions on neural networks, vol. 6, no. 3, may 1995. 